\title{
Enfoque alimentario desde la atención primaria de salud
}

\section{Food approach from primary health care}

\author{
Diana Laura Guerra Ortega ${ }^{1 *}$, Elizabeth Marrero Escalona ${ }^{1}$, Sara Elizabeth Bravo Salinas ${ }^{1}$ \\ ${ }^{1}$ Universidad Católica de Cuenca \\ *diana.guerra@ucacue.edu.ec
}

DOI: https://doi.org/10.26871/killkana_salud.v3i3.524

\begin{abstract}
Resumen
Contexto: La contribución en el mantenimiento de la salud desde la atención primaria permite disminuir la aparición temprana de enfermedades prevenibles, como las relacionadas con malos hábitos de alimentación y de actividad física que se han incrementado en la población, convirtiéndose en un problema de salud pública en la actualidad. Objetivo: Brindar información y conocimientos sobre la alimentación enfocada al ciclo vital de cada individuo según su desarrollo, para apoyar a los servidores de atención primaria con algunos lineamientos alimentarios. Procedimiento: Mediante la exploración de bases de datos en buscadores médicos se evalúan y seleccionan artículos con evidencia científica en cuanto a las guías anticipatorias alimentarias en todos los ciclos de la vida del individuo. Resultados: La implementación de alimentos adecuados desde el inicio de la vida de un ser humano al igual que la modificación de hábitos durante el desarrollo, reducen notablemente la aparición de enfermedades relacionadas con malnutrición, teniendo un mayor impacto en la disminución de obesidad y desnutrición en las etapas más vulnerables de la vida que son los niños, además se ha evidenciado efectos positivos de la alimentación saludable en adultos jóvenes y mayores con la disminución de aparición de enfermedades cardiovasculares y metabólicas. Conclusiones: La alimentación saludable es fuente de vida en cualquier etapa del desarrollo de un individuo, así, desde edades tempranas se pueden ir adquiriendo y modificando dichos hábitos, mientras que en la adultez generalmente ya tenemos patrones alimentarios establecidos, pero podemos lograr cambios positivos para mejorar hábitos de alimentación saludable y disminuir la mobimortalidad.
\end{abstract}

Palabras clave: alimentación, atención primaria, salud.

\begin{abstract}
Context: The contribution to the maintenance of health from primary care allows to reduce the early onset of preventable diseases, such as those related to bad eating habits and physical activity that have increased in the population, becoming a Public health problem today. Objective: Provide information and knowledge about food focused on the life cycle of each individual according to their development, to support primary care servers with some food guidelines. Procedure: Through the search of databases in medical search engines, articles with scientific evidence regarding the anticipatory dietary guidelines in all life cycles of the individual are evaluated and selected. Results: The implementation of adequate food from the beginning of the life of a human being as well as the modification of habits during development, significantly reduce the occurrence of diseases related to malnutrition, having a greater impact on the decrease of Obesity and malnutrition in the most vulnerable stages of life that are children, in addition there have been positive effects of healthy eating in young and old adults with the decrease in the appearance of cardiovascular and metabolic diseases. Conclusions: Healthy eating is a source of life at any stage of the development of an individual, so from an early age these habits can be acquired and modified, while in adulthood we usually already have established eating patterns, but we can achieve positive changes to improve healthy eating habits and decrease mobility.
\end{abstract}

Keywords: eating, primary attention, health .

\section{Introducción}

La salud no es solo el estado de ausencia de enfermedades, es más bien un proceso de bienestar físico y psicosocial que hay que conservarlo y contribuir a su mantenimiento mediante medidas preventivas. En los servicios de salud están efectuados mediante la atención primaria que es la que representa el primer contacto que tienen los individuos con los servicios nacionales de salud, lo que constituye una estrategia que permite garantizar la atención a toda la comunidad y que tengan los recursos necesarios para una vida sana y con dignidad. ${ }^{1}$

Hoy en día, a nivel mundial, las enfermedades relacionadas con los malos hábitos de alimentación y de actividad física, se encuentran en tasas crecientes y forman parte del principal factor de riesgo para desarrollar enfermedades no trasmisibles como enfermedades cardiovasculares, hipertensión arterial, diabetes mellitus tipo 2, obesidad, sobrepeso, osteoporosis y trastornos psicosociales. Esta situación 
afecta con mayor impacto a comunidades con poca accesibilidad a centros de salud y de bajos recursos económicos, lo que como consecuencia la afectación de poblaciones más vulnerables como los niños. ${ }^{2}$ En países menos desarrollos como señala la Organización Mundial de la Salud (OMS), los malos hábitos alimenticios vinculados con ingestión de alimentos contaminados con microorganismos se relacionan con enfermedades trasmitidas por los alimentos, en especifico en un $70 \%$ las diarreas son causadas por bacterias, hongos, virus, parásitos, toxinas y metales pesados. Por esta razón es que el médico especialista en atención primaria deberá enfrentarlo y tener conocimientos claros sobre guías anticipatorias alimentarias para los individuos, independientemente del ciclo vital de cada individuo ya sean niños, adolescentes, adultos y adultos mayores. ${ }^{3,4}$

Un adecuado proceso de alimentación en la población permite que el individuo se desarrolle de una manera óptima, siendo de mucha importancia que cada persona adquiera educación sobre como tener hábitos alimenticios adecuados, los mismos que en etapas tempranas de la vida va dirigida hacia los padres o cuidadores de neonatos y lactantes, estimándose que a partir de los preescolares se inicia un proceso en el que se pueden adquirir hábitos saludables de vida ya que presenta autonomía y conciencia sobre la salud. ${ }^{5}$

El objetivo de conocer sobre la alimentación es para apoyar a los servidores de atención primaria que están en contacto con la comunidad, con los lineamientos básicos en cuanto a parámetros alimentarios a considerarse con la atención de cada miembro de la familia.

Metodología: mediante la exploración de bases de datos en buscadores médicos como pubmed, trip database, clinicalkey, uptodate, elsevier se evalúan y seleccionan artículos con evidencia científica en español e inglés publicados desde el 2014 en cuanto a las guías anticipatorias alimentarias en todos los ciclos de la vida de un individuo.

\section{Desarrollo y Discusión}

En los centros nacionales de salud, el Médico Familiar es el efector de la atención primaria que brinda a los individuos y a toda la comunidad estrategias de promoción, prevención y de tratamiento para los padecimientos con mayor presentación en la comunidad, en consideración con que el aparecimiento de enfermedades no trasmisibles y crónicas por malos hábitos alimenticios y físicos han salido a relucir en los últimos tiempos, es prioritaria la educación en cuanto a la dieta, que constituye una medida básica y simple que todos los individuos pueden cumplirla en dependencia del ciclo vital en que se encuentren. A continuación, se detallan algunos aspectos relevantes sobre el manejo de la alimentación en la población en general, sin hacer detalle en la población que presenta comorbilidades.

\subsection{Neonatos}

Esta etapa comprende al recién nacido hasta los 28 días de vida. Es el período de mayor vulnerabilidad durante las
24 horas de vida, donde se presentan muertes neonatales de diversas causas, en un $70 \%$ son prevenibles mediante medidas de costo efectivas, entre ellas el mantenimiento de la alimentación con leche materna, mediante educación continua con la madre, más aún en lugares de bajos recursos económicos. ${ }^{6,7}$

La nutrición del neonato abarca también la conservación de la salud de la madre para que la leche materna esté en óptimas condiciones. Hay que tener en cuenta que el volumen de la leche materna puede variar y que los nutrientes de la misma provienen de la alimentación materna o de las provisiones nutricionales de la madre. ${ }^{8}$

Para la madre es necesario establecer los tiempos en los que se debe administrar la leche materna, y hasta cuándo es el momento idóneo para complementar la alimentación del recién nacido. En esta etapa la alimentación se basa mediante leche materna exclusiva a libre demanda, sin el consumo de otro alimento o líquido, según la Organización Mundial de la Salud (OMS) se prolonga hasta los 6 meses de vida, tiempos más cortos de lactancia exclusiva se han relacionado con aparición de obesidad en edades tempranas según varios estudios. ${ }^{8-11}$ Sin embargo, la existencia de condiciones maternas o neonatales que dificulten la lactancia exclusiva, hace que sea necesario el incremento de fórmulas infantiles a la dieta del recién nacido, sin dejar de lado la educación de forma correcta para dar de lactar hasta que exista el apego adecuado, con el único objetivo de conservar y continuar con el desarrollo y crecimiento adecuado del niño. Para el efecto, las fórmulas de inicio son las que cubren las demandas nutricionales del recién nacido y lactantes hasta los 4 y 6 meses de edad, y las fórmulas de continuación a partir de los 4 a 6 meses. ${ }^{12,13}$ Las fórmulas de inicio proporcionan energía, aporte proteico, graso y otros nutrientes, que a medida que avanza el desarrollo deben ser regulados ya que relacionan con aumento del riesgo de obesidad a largo plazo. ${ }^{12}$ Los requerimientos energéticos de los neonatos y hasta los dos meses de edad son de 100 a $110 \mathrm{kcal} / \mathrm{kg}$ por día, y van a variar considerablemente con las necesidades metabólicas de cada individuo y de sus características individuales, las cuales deben tomar en consideración para evitar complicaciones gastrointestinales. ${ }^{14}$

En conjunto con la educación alimentaria se evalúan las habilidades del neonato logradas con la lactancia, que condicionan luego el buen apego a la alimentación complementaria. Estas habilidades alimentarias en el recién nacido son la succión, deglución, reflejo de búsqueda y reflejo de extrusión de la lengua, relacionadas con el desarrollo cognitivo y psicomotor que alertarían a la presencia de comorbilidades. ${ }^{9,15}$

\subsection{Lactantes}

Comprende a partir de los 28 días hasta los 24 meses de edad. Son lactantes menores desde los 28 días hasta el primer año de vida, y lactantes mayores desde 1 año hasta los 2 años de vida. En lactantes la alimentación es 
crucial, e implica el conocimiento del rápido crecimiento y requerimiento de nutrientes y energía necesarias para el óptimo desarrollo de todo su potencial a corto, mediano y largo plazo, así se considera como un alimento de elección durante los primeros 6 meses de vida a la leche materna. ${ }^{16-18}$

Esta es una etapa de mayor riesgo de presentar trastornos relacionados con deficiencias nutricionales de micronutrientes y enfermedades infecciosas como la diarrea de origen viral, que tiene como consecuencias la desaceleración en el crecimiento y desarrollo, desnutrición a corto y mediano plazo, y a largo plazo en la adolescencia y adultez presentan problemas relacionados con menor capacidad y rendimiento intelectual, y que condiciona a la programación metabólica temprana con el exceso o déficit de nutrientes para el posterior desarrollo de enfermedades metabólicas crónicas en el adulto. ${ }^{9,19}$

\subsubsection{Alimentación complementaria}

"Es la introducción de alimentos sólidos y líquidos incluyendo las fórmulas infantiles en la dieta del niño" según la OMS, siendo considerada por esta entidad a partir de los 6 meses. Sin embargo, Duryea manifiesta que en dependencia de la maduración del desarrollo neurológico, la alimentación complementaria óptima puede ser desde el cuarto al sexto mes de vida. ${ }^{14,20,21}$ Los requerimientos energéticos en los lactantes desde los 3 meses hasta los 11 meses varían desde 80 a $95 \mathrm{kcal} / \mathrm{kg}$ por día. Los alimentos complementarios están influenciados por la cultura de cada comunidad, existen algunas directrices de la Sociedad Europea de Gastroenterología, Hepatología y Nutrición Pediátrica y de los Comités de Nutrición de la Academia Americana de Pediatría, que indican que la introducción de sólidos a la dieta debe ser gradual con la leche materna o fórmula infantiles hasta ir reduciéndolo paulatinamente a 28 o 32 onzas por día. ${ }^{14}$

Se recomienda que los alimentos de un solo ingrediente deben ser administrados primero, luego de su tolerancia se introduce a la dieta las frutas y verduras al menos una vez al día a manera de coladas o puré. Es el arroz un cereal que se ofrece con mayor frecuencia, y primero por su fácil acceso y por sus propiedades menos alergénicas, además, es necesario que al menos una comida tenga contenido de vitamina $\mathrm{C}$ para una adecuada absorción del hierro, la colocación de sal o azúcar a los alimentos no se han relacionado con mayor aceptación de la comida por el niño. ${ }^{10,14}$ Deben evitarse los alimentos duros y redondos porque pueden provocar asfixia, leche entera de vaca por mayor contenido de solutos renales antes de los 12 meses, y las bebidas azucaradas porque se relacionan con riesgo de obesidad a los 6 años. ${ }^{14,22}$

\subsection{Niñez: Preescolares y Escolares}

En este ciclo vital -que está comprendido a partir de los 3 a 5 años, conociéndose como preescolares y desde los 6 a 12 años, como escolares- la alimentación debe basarse de acuerdo al conocimiento de las características de crecimiento de cada individuo, al igual que de su hábito alimentario. En la edad preescolar se está frente a un crecimiento estable, alcanzándose un incremento ponderal anual de 2 a $3 \mathrm{~kg}$, mientras que en los escolares es un crecimiento un poco más lento y el peso aumenta de 3 a 3,5 $\mathrm{kg}$ anuales en conjunto con la maduración de sus aparatos y sistemas. ${ }^{23}$

En la edad preescolar los niños empiezan a elegir los alimentos y la mayoría tiene preferencia por alimentos no saludables (papas fritas, pizzas, leche con sabor, etc); por lo que tiene importancia la observación y la imitación en donde es fundamental cultivar buenos hábitos alimentarios a través del modelamiento del comportamiento, mientras que la alimentación en escolares se hace más independiente del entorno familiar. ${ }^{23-26}$

Los requerimientos nutricionales en la niñez dependen de las demandas metabólicas basales, de la actividad física, del desarrollo psicológico y sexo. La ingesta de energía puede estar determinada por la cantidad de comidas y refrigerios durante el día, los padres o cuidadores deben proporcionar los alimentos nutritivos, pero se debe respetar la decisión del niño en cuanto a la cantidad que desea comer. La alimentación en la niñez se basa en proporcionar energía y micronutrientes que incluyen vitaminas, minerales y oligoelementos, la energía se deriva de proteínas, grasas y carbohidratos. ${ }^{27}$

La frecuencia diaria alimentaria recomendada es de 4 a 6 veces, regulando la porción que dependerá de la edad y del tipo de comida a ingerirse. Se considera que los suplementos de vitaminas y minerales no son necesarios para los niños en los que se evidencia un crecimiento sano y que consumen una dieta adecuada y variada. Para minimizar las enfermedades trasmitidas por los alimentos, por lo general las infecciosas como la diarrea, los alimentos deben ser pasteurizados, y deben evitarse los que son crudos como el huevo, el marisco, la carne, y el pollo. ${ }^{24}$

En esta etapa la ingesta inadecuada de nutrientes puede condicionar un déficit de peso del niño que implica consecuencias en su desarrollo y crecimiento, o un aumento de peso que produzca obesidad y con ello predisponer en su etapa de adultez enfermedades cardiovasculares, por lo que el médico debe evaluar la existencia de enfermedad orgánica o problemas psicosociales o ambientales. ${ }^{28,29}$

\subsection{Adolescentes}

Según la OMS, la alimentación es concluyente en la prevención de enfermedades crónicas mediante la implementación de hábitos alimentarios adecuados. Sin embargo, se han identificado hábitos que se caracterizan por un consumo excesivo de nutrientes, por un tipo de dieta rica en proteínas y grasas de origen animal, situación en la que están inmersos los adolescentes. Esto ha determinado la aparición de patología propias del adulto a edades más tempranas, como la diabetes tipo 2, hígado graso, o apnea 
del sueño, las mismas que están muy ligadas a la mala alimentación. . $^{28,30,31}$

Datos estadísticos revelan que en España un porcentaje de adolescentes entre 12 a 17 años presentan obesidad abdominal, que va acompañado a un mayor riesgo cardiometabólico; además se han observado otros trastornos asociados a obesidad como trastornos de la conducta y el aprendizaje, que incluye estados depresivos en el adolescente. También son más propensos a problemas de articulaciones, alergias y cefaleas. ${ }^{31,32}$

La alimentación en la adolescencia tiene una enorme transcendencia en la normativa de vida de la adultez. En la adolescencia se fortalecen hábitos de alimentación que pueden ser favorables o dañinos a la salud. Por eso es importante llevar una dieta apropiada en este ciclo de la vida, que a su vez marcará la dieta del adulto determinando en gran medida el proceso futuro de salud-enfermedad del individuo en desarrollo.

A la adolescencia se le caracteriza como un periodo vulnerable en el desarrollo de un individuo ya que implica muchos cambios en el ciclo vital. ${ }^{32}$ En esta etapa los hábitos que se adquieran serán los que perduren en la vida adulta y serán concluyentes en los estados de salud, tanto protectores como contribuyentes a la formación de factores de riesgo para desarrollar enfermedades. ${ }^{33}$

La alimentación es fundamental en esta etapa sobre todo para cubrir las demandas energéticas que requiere el desarrollo ideal del organismo, también se depende de las condiciones de cada individuo, como por ejemplo: el sexo, la actividad física, entre otras. ${ }^{31,32}$

Palenzuela Paniagua y colaboradores encontraron en un estudio sobre la alimentación en adolescentes que hay frecuencias incrementadas del consumo de alimentos poco saludables, en específico en poblaciones de condiciones sociales bajas y determinan que los hábitos alimentarios en adolescentes incumplen con los estándares de comida saludable. Esto coincide con otros estudios a que esta situación se debe a la relación del nivel de educación alcanzada por la madre y el nieve socioeconómico bajo familiar, con mayor ingesta de alimentos poco nutritivos. ${ }^{30}$

Miqueleiz en su estudio establece causas de la situación poco apropiada de los hábitos alimentarios en adolescentes hace énfasis en la relación de las condiciones socioeconómicas en las que se encuentre el individuo, así los adolescentes pertenecientes a familias de posición socioeconómica limitada, tienen características alimentarias poco saludables más frecuentemente que los que pertenecen a familias de buena posición económica. Se corroboran estos resultados con los obtenidos en investigaciones en otros países como Francia, Finlandia y Dinamarca. ${ }^{34}$

En esta etapa la alimentación debe tener algunas características que representen ser saludables y que ayuden al mantenimiento de la salud del individuo adolescente, en dependencia del requerimiento energético de cada individuo y mediante la conservación de principios básicos el momento de alimentarse, como son mantener en equilibrio la variedad de alimentación para consumirla con moderación. ${ }^{35}$

Un adolescente incluye en su dieta diaria alimentos con cantidades disminuidas de frutas, cereales y verduras. Se ha evidenciado a través de estudios a adolescentes españoles, que hay un consumo inferior de productos lácteos, frutas y vegetales, en comparación con un consumo alto de alimentos con deficiente aporte nutricional. ${ }^{32}$

Se determinóque en los adolescentes de sexo masculino hay mayores requerimientos nutricionales que en la mujer, estableciéndose que los varones requieren entre 1.800 y $3.200 \mathrm{kcal}$, mientras que la mujer adolescente necesita entre 1.600 y $2.400 \mathrm{kcal}$, sin embargo, existen variaciones de los requerimientos en función de la actividad física que realice cada individuo. Parte de los requerimientos nutricionales de un adolescente lo constituyen los hidratos de carbono y van a variar con el crecimiento al igual que las necesidades de las proteínas. ${ }^{32}$

Lo más importante es enseñar a los adolecentes a saber qué tipos de alimentos deben ingerir, más que los que deben evitar. Así, la adolescencia es un estado idóneo para enseñar hábitos alimentarios saludables, quizás es de las últimas etapas de la vida en la que se puede modificar la alimentación del individuo; es la oportunidad de actuar y obtener adultos sanos en el futuro.

\subsection{Adultez}

La alimentación en el adulto es fundamental en la determinación del estado de salud del individuo y repercute de manera directa en este. Si se consume exceso de grasas saturadas y/o carbohidratos, aumenta el riesgo de dislipidemias que constituyen patologías muy asociadas a eventos cardiovasculares y cerebrovasculares, al igual que a cardiopatías, diabetes y cáncer. ${ }^{36}$

Además, se asocian a la aterosclerosis, entidad muy relacionada a complicaciones diversas. Así mismo una dieta rica en azúcares y el sedentarismo asociado al sobrepeso, aumenta el riesgo de padecer diabetes mellitus, enfermedad que en la actualidad afecta a muchas personas a nivel mundial. Por ende, la importancia de una alimentación saludable se impone. ${ }^{28,37-39}$

Se han realizado múltiples estudios que relacionan la dieta equilibrada con la salud, por lo que es conocido que los buenos hábitos alimentarios evitan la aparición temprana de enfermedades de tipo metabólico. ${ }^{37,40,41}$ Esto ha quedado demostrado y no se cuestiona su veracidad.

La alimentación puede actuar como un desencadenante de enfermedad o como protector de la misma en relación a las enfermedades cardiovasculares, diferentes tipos de cáncer, diabetes, osteoporosis, entre otras patologías. ${ }^{32}$

En estudio que se desarrolló en España, con Blázquez y colaboradores, encontraron en cuanto a alimentación que la población de estudio presentaba niveles de alimentación poco saludable con relación a la ingesta de vegetales, carne y mucho más bajo a frutas, también de manera general se observó un escaso cumplimiento de las recomendaciones 
para la administración diaria de alimentos que se encuentran ubicados al inicio de la pirámide de la alimentación. Muy similar resultado se obtuvo en la Encuesta Nacional de Ingesta Dietética, llevada a cabo por la Agencia Española de Consumo, Seguridad Alimentaria y Nutrición. ${ }^{40}$ Otros estudios como los realizados en Chile, así como las Encuestas Nacionales de Salud y de Consumo Alimentario muestran que una gran cantidad de la población presenta un bajo consumo de frutas, verduras, lácteos, pescado y leguminosas y opuestamente un consumo alto de alimentos no saludables con gran contenido de energía, grasas, azúcar y sodio. ${ }^{41}$ Estudios similares realizados en diferentes instituciones universitarias, dan a relucir resultados que se relacionan a malos hábitos de alimentación, en específico en cuanto al consumo deficiente de verduras y frutas, en relación con un aumento del consumo de golosinas. ${ }^{42}$

Cabe recalcar la importancia del consumo de alimentos considerados saludables como vegetales, legumbres y frutas, al igual que evitar el exceso de grasas y azúcares es una buena medida para comenzar a modificar el tipo de vida en el adulto. Las comidas en pocas cantidades y fraccionadas en el día son recomendadas hoy en día. Si bien es conocido que cuando se llega a la adultez ya están formados los patrones de alimentación y resulta más complejo modificarlos, no es imposible cambiarlos para mejor, en aras de preservar la salud y evitar enfermedades prevenibles de esta manera y en caso que aparezcan evitar sus complicaciones. ${ }^{43,44}$

\subsection{Adulto Mayor}

Los trastornos que se relacionan con el aumento de peso como la obesidad son problemas de salud pública bien establecidos y que cada vez más afecta a edades extremas de la vida como son la niñez y adultos mayores, hecho que, acompañado al poco conocimiento de estilos de alimentación saludable, supone mayor riesgo por muerte cardiovascular futura. ${ }^{40,45} \mathrm{En}$ el adulto mayor las enfermedades cardiovasculares aumentan el riesgo de caídas que pueden llegar tener secuelas graves en el individuo, desde invalidez hasta la muerte. Además, se pueden convertir en un problema para la familia y la sociedad que son encargados de velar por la seguridad y bienestar de sus ancianos. $^{46-48}$

Valdés Badilla et al, en su estudio en España encontraron entre sus principales resultados que la ingesta de algunos alimentos pueden contribuir a ser factores protectores y generadores de obesidad, entre estos se destacan que las frutas son protectoras frente a la obesidad sumándose además, un consumo diario de agua que actúa como protector, mientras que los que representaron ser favorecedores para obesidad fueron los snacks, que incrementan la adiposidad en el abdomen y aumenta el riesgo cardiovascular, también la ingesta de alcohol se relaciona con esto. Los mismos concluyen que los adultos mayores que presentan mayor frecuencia en el consumo de alimentos que se consideran como saludables, disminuyen notablemente el riesgo car- diovascular, al igual que la grasa abdominal en comparación con los adultos mayores que ingieren alimentación poco saludable que presentaron mal estado nutricional. Este estudio demuestra la necesidad de una dieta sana en la tercera edad, que disminuye de forma significativa el riesgo de morbilidades. ${ }^{40}$

En el adulto mayor por lo general se encuentran establecidos ciertos hábitos de alimentación más variada en comparación con la población más joven. Sin embargo, predominan algunos factores que condicionan el consumo de alimentación saludable, como el estado físico y recursos económicos que influyen en los estados psicosociales de cada individuo, interviniendo en el hábito de la alimentación con respecto a calidad y cantidad de alimentos que adquieren que muchas veces no son adecuados. Esto trae como consecuencia que aumente el riesgo de morbilidad y mortalidad, además de contribuir con estados de enfermedades crónicas e infecciones asociadas con el envejecimiento, que por lo general disminuyen una buena calidad de vida a las personas que se encuentran en este proceso natural de la vida, caso contrario con una construcción adecuada de alimentación se prolonga la calidad de vida y se mantiene la salud. ${ }^{49}$

Centers for Disease Control and Prevention (CDC), señala que "la longevidad depende en $19 \%$ de la genética, en un $10 \%$ del acceso a atención médica, $20 \%$ de factores ambientales como la contaminación y $51 \%$ de los factores de estilos de vida". ${ }^{49}$

Barrón y colaboradores, encontraron en su estudio en Chile que una gran cantidad de adultos mayores acostumbran a ingerir tres comidas diarias que comprenden un desayuno, un almuerzo y una comida durante la noche; y que en cada plato se coloca una a dos porciones de lácteos y alimentos bajos en grasa. Además, las ingestas relacionadas con el calcio contribuyen al mantenimiento de la salud ósea, mientras que la baja ingesta de calcio aumenta el riesgo de facturas ya que este y la vitamina $\mathrm{D}$ son indispensables para prevenir la osteoporosis y la reducción ósea en los últimos años de vida. Se recomienda que el consumo de verduras y frutas en esta etapa incrementa el aporte de fibra para mejorar el metabolismo y las condiciones fisiológicas de cada individuo. Coinciden otros estudios con el consumo beneficioso de fibra soluble de 3 a 10 gr/día, que baja de forma proporcional el colesterol total sanguíneo y las concentraciones de LDL que es considerado el colesterol malo debido a su asociación a eventos cardiovasculares adversos cuando aumentan sus niveles. ${ }^{47,49}$

Los adultos mayores constituyen un grupo vulnerable, donde hay un incremento de la prevalencia de las enfermedades crónicas no transmisibles. Es bien conocido como una alimentación saludable, rica en frutas, vegetales, legumbres y la adecuada cantidad de lácteos pueden ayudar en la prevención. Es importante el cuidado de los ancianos para que puedan tener acceso a estos alimentos y cuenten con una dieta balanceada, garantizado de esta manera una longevidad satisfactoria, con calidad de vida, donde conser- 
ven su autonomía e independencia. El envejecimiento sin la presencia de enfermedades, depende de muchos factores como la alimentación adecuada y equilibrada que jugará un papel muy importante de acuerdo a la OMS..$^{50}$

\section{Conclusiones}

La alimentación saludable es fuente de vida en cualquier etapa del desarrollo de un individuo. A la vez una dieta inadecuada está acompañada de morbimortalidad demostrada, sobre todo en la adultez y en la tercera edad, por ello es importante desde etapas tempranas inculcar hábitos saludables de vida en cuanto a la alimentación.

En las primeras épocas de la vida se inicia con la educación a toda la familia, primeramente, con la madre en la etapa de lactancia y posteriormente a los miembros de la familia para que adquieran los hábitos saludables en cada ciclo vital en que se encuentren, así a partir de la edad preescolar conjuntamente con la autonomía que adquiere el niño se deben iniciar las pautas de una alimentación saludable a partir la observación e imitación del niño permitiendo la modificación de sus hábitos.

Posteriormente con el crecimiento del niño, es adecuado mantener claras las frecuencias y porciones necesarias de alimentos que permitan cubrir las necesidades metabólicas basales y evitar los excesos que condicionarían a la presencia de enfermedades a largo plazo. La adolescencia es posiblemente la última etapa más importante en la adquisición de hábitos de alimentación saludable, en la que aún podemos lograr incorporar alimentos que ayuden a un desarrollo físico y psíquico adecuado.

En la adultez generalmente ya tenemos patrones alimentarios establecidos, pero podemos lograr modificaciones positivas en casos que sean necesarios y adquirir hábitos de alimentación saludable. Los adultos mayores precisan nuestra atención especial para garantizar en ellos una dieta sana y de esta manera disminuir el riesgo de enfermedades, caídas y fracturas; elementos que aportan como causas de muerte en este grupo de edad. Fomentar políticas de 10 . educación en términos de alimentación en todas las etapas de la vida es fundamental, inculcar una dieta rica en frutas, verduras, legumbres, lácteos, proteínas; balanceada y variada es clave importante en el mantenimiento del estado de 11. salud. De igual manera disminuir el consumo excesivo de grasas y azúcares. Una dieta sana garantiza una vida plena y con calidad.

\section{Fuente de Financiamiento}

Este estudio es autofinanciado

\section{Conflicto de Intereses}

No existen conflictos personales, profesionales, financieroso de otro tipo.

\section{Consentimiento Informado}

Los autores cuentan con el consentimiento informado de 14 los paciente para la investigación, la publicación del caso y sus imágenes.

\section{Referencias Bibliográficas}

1. Organización Mundial de la Salud. ¿Cómo define la OMS la Salud?; 2019.

2. Ricardo YR, Peralta L, Yaulema L, Pallo J, Orozco D, Caiza V, et al. Alimentación saludable en docentes. Revista Cubana de Medicina General Integral. 2017 may;33(1).

3. World Health Organization. OMS I Estimaciones de la OMS sobre la carga mundial de enfermedades de transmisión alimentaria; 2015.

4. Novales M, Galván Melendez M, Flores Soto N, Morales Castro M, álvarez Meraz S, Reyes K, et al. Revista Enfermedades Infecciosas y Microbología \#2 2019. 2019 jun;39(2):33.

5. Rodríguez RA. Alimentación del niño en edad preescolar. Importancia de la educación para la salud. NURE investigación: Revista Científica de enfermería. 2018;15(94 (Junio-Julio 2018)):7.

6. Agudelo SI, Maldonado MJ, Orozco C, Restrepo J, Gamboa $\mathrm{O}$. Efecto de un sistema de enseñanza personalizado en la evaluación de competencias en el manejo del neonato. Revista Cubana de Educación Médica Superior. 2015 dec;29(4):725-741.

7. Lean RE, Smyser CD, Rogers C. Assessment: The Newborn. Child and adolescent psychiatric clinics of North America. 2017 jul;26(3):427-440.

8. Ares Segura S, Arena Ansótegui J, Díaz-Gómez NM. La importancia de la nutrición materna durante la lactancia, ¿necesitan las madres lactantes suplementos nutricionales? Anales de Pediatría. 2016 jun;84(6):347.e1-347.e7.

9. Romero-Velarde E, Villalpando-Carrión S, Pérez-Lizaur AB, Iracheta-Gerez MdlL, Alonso-Rivera CG, LópezNavarrete GE, et al. Consenso para las prácticas de alimentación complementaria en lactantes sanos. Boletín Médico del Hospital Infantil de México. 2016 sep;73(5):338-356.

10. Sandoval Jurado L, Jiménez Báez MV, Olivares Juárez S, de la Cruz Olvera T. Lactancia materna, alimentación complementaria y el riesgo de obesidad infantil. Atención Primaria. 2016 nov;48(9):572-578.

11. Toscano N, facebook com/pahowho H. OPS/OMS I Semana Mundial de la Lactancia Materna 2018: Pilar de la vida; 2018.

12. Pedrón Giner C, Cuervas-Mons Vendrell M, Galera Martínez R, Gómez López L, Gomis Muñoz P, IrastorzaTerradillos I, et al. Guía de práctica clínica SENPE/SEGHNP/SEFH sobre nutrición parenteral pediátrica. Nutrición Hospitalaria. 2017 jun;34(3):745-758.

13. Kellams A, Harrel C, Omage S, Gregory C, Rosen-Carole C. ABM Clinical Protocol \#3: Supplementary Feedings in the Healthy Term Breastfed Neonate, Revised 2017. Breastfeeding Medicine: The Official Journal of the Academy of Breastfeeding Medicine. 2017;12:188-198.

. Duryea T, Drutz J, Motil K, Torchia M. Introducing solid foods and vitamin and mineral supplementation during infancy - UpToDate. 2019 aug;. 
15. Sudarshan J, Abrams S. Neonatal oral feeding difficulties 30. Palenzuela Paniagua SM, Pérez Milena A, Torres LAPd, due to sucking and swallowing disorders - UpToDate; 2019.

16. World Health Organization. OMS I Lactante, recién nacido; 2019.

17. Zurro AM, Pérez JFC, Badia JG. Atención primaria. Problemas de salud en la consulta de medicina de familia. Elsevier Health Sciences; 2019.

18. Marchand V. The toddler who is falling off the growth chart. Paediatrics \& Child Health. 2014 oct;17(8):447450 .

19. Harris JB, Pietroni M. Approach to the child with acute diarrhea in resourcelimited countries. Agosto de 2016. 2016 mar;

20. World Health Organization. WHO I Protecting, promoting, and supporting breastfeeding in facilities providing ma-34. Miqueleiz E, Lostao L, Ortega P, Santos JM, Astasio P, ternity and newborn services: the revised Baby-friendly Hospital Initiative 2018; 2018.

21. Cuadros-Mendoza CA, Vichido-Luna MA, MontijoBarrios E, Zárate-Mondragón F, Cadena-León JF, 35. Vilaplana i Batalla M. Hábitos alimentarios. Farmacia Cervantes-Bustamante R, et al. Actualidades en Profesional. 2016 mar;30(2):15-18. alimentación complementaria. Acta pediátrica de 36. Organización Mundial de la Salud. Malnutrición; 2019. México. 2017 jun;38(3):182-201.

37. Vilaplana i Batalla M. Nutrición y diabetes. Farmacia Profesional. 2015 mar;29(2):26-30.

22. Espín Jaime B, Díaz Martín JJ, Blesa Baviera LC, Claver Monzón n, Hernández Hernández A, García Burriel JI, 38 et al. Alergia a las proteínas de leche de vaca no mediada por IgE: documento de consenso de la Sociedad Española de Gastroenterología, Hepatología y Nutrición Pediátrica (SEGHNP), la Asociación Española de Pediatría de Aten- 39. ción Primaria (AEPAP), la Sociedad Española de Pediatría Extrahospitalaria y Atención Primaria (SEPEAP) y la Sociedad Española de Inmunología Clínica, Alergología y Asma Pediátrica (SEICAP). Anales de Pediatría. 201940. mar;90(3):193.e1-193.e11.

23. Moreno Villares JM, Galiano Segovia MJ. Alimentación del niño preescolar, escolar y del adolescente. 2015 may;XIX:10.

24. Duryea TK, Drutz JE, Motil K, Torchia M. Dietary recommendations for toddlers, preschool, and school-age children - UpToDate; 2019.

25. Campos Rivera NH, Lagunes IR. Preferencias Alimentarias y su Asociación con Alimentos Saludables y No Saludables en Niños Preescolares. Acta de Investigación Psicológica. 2014 apr;4(1):1385-1397.

Fernández García JA, Maldonado Alconada J. La alimentación en el adolescente. Anales del Sistema Sanitario de Navarra. 2014 apr;37(1):47-58.

31. Martí A, Martínez JA. La alimentación del adolescente: necesidad imperiosa de actuar de forma inmediata. Anales del Sistema Sanitario de Navarra. 2014 apr;37(1):5-8.

32. Morales C, Andrés E. Impacto de la obesidad en la salud de la niña y de la adolescente. Revista Peruana de Ginecología y Obstetricia. 2017 oct;63(4):599-606.

33. Alfaro González M, Vázquez Fernández ME, Fierro Urturi A, Rodríguez Molinero L, Muñoz Moreno $\mathrm{M}^{\mathrm{a}} \mathrm{F}$, Herrero Bregón B, et al. Hábitos de alimentación y ejercicio físico en los adolescentes. Pediatría Atención Primaria. 2016 sep;18(71):221-229.

Regidor E. Patrón socioeconómico en la alimentación no saludable en niños y adolescentes en España. Atención Primaria. 2014 oct;46(8):433-439.

. Pallarés-Carratalá V, Pascual-Fuster V, Godoy-Rocatí D. Dislipidemia y riesgo vascular. Una revisión basada en nuevas evidencias. Medicina de Familia SEMERGEN. 2015 nov;41(8):435-445.

9. Sociedad Europea de Cardiología (ESC), European Atherosclerosis Society (EAS). Guía ESC/EAS 2016 sobre el tratamiento de las dislipemias. Revista Española de Cardiología. 2017 feb;70(2):115.e1-115.e64.

. Blázquez Abellán G, López-Torres Hidalgo JD, Rabanales Sotos J, López-Torres López J, Val Jiménez CL. Alimentación saludable y autopercepción de salud. Atención Primaria. 2016 oct;48(8):535-542.

41. Ratner R, Hernández P, Martel J, Atalah E. Propuesta de un nuevo índice de calidad global de la alimentación. Revista chilena de nutrición. 2017 mar;44(1):33-38.

42. Becerra-Bulla F, Pinzón-Villate G, Vargas-Zárate M. Practicas alimentarias de un grupo de estudiantes universitarios y las dificultades percibidas para realizar una alimentación saludable. Revista de la Facultad de Medicina. 2015 jul;63(3):457-453.

26. Sánchez-García R, Reyes-Morales H, González-Unzaga 43. Berciano S, Ordovás JM. Nutrición y salud carMA. Preferencias alimentarias y estado de nutrición en niños escolares de la Ciudad de México. Boletín Médico del Hospital Infantil de México. 2014 nov;71(6):358-366. 44.

27. USDA. Dietary Reference Intakes I Food and Nutrition Information Center I NAL I USDA; 2019.

diovascular. Revista Española de Cardiología. 2014 sep;67(09):738-747.

4. Sociedad Española de Nutrición Comunitaria. La pirámide nutricional: educación en nutrición; 2019.

28. García Milian AJ, Creus García ED. La obesidad como factor de riesgo, sus determinantes y tratamiento. Revista Cubana de Medicina General Integral. 2016 sep;32(3):00 .

45. Gómez Sanchiz M, Martínez Checa J, Muñoz Blázquez C, Valenzuela Pulido N. Alimentación y hábitos saludables en una población mayor. Medicina General y de Familia. 2016 jan;5(1):3-8.

46. Machado Cuétara RL, Bazán Machado MA, Izaguirre Bordelois M. Principales factores de riesgo asociados a las caídas en ancianos del área de salud Guanabo. 
MEDISAN. 2014 feb;18(2):158-164.

47. Alvarado-García A, Lamprea-Reyes L, Murcia-Tabares K. La nutrición en el adulto mayor: una oportunidad para el cuidado de enfermería. Enfermería Universitaria. 2017 jul;14(3):199-206.

48. Vilaplana i Batalla M. Alimentación en el anciano. Farmacia Profesional. 2017 jan;31(1):17-20.

49. Barrón V, Rodríguez A, Chavarría P. Hábitos alimentarios, estado nutricional y estilos de vida en adultos mayores activos de la ciudad de Chillán, Chile. Revista chilena de nutrición. 2017 mar;44(1):57-62.

50. Organización Mundial de la Salud. OMS । Datos interesantes acerca del envejecimiento; 2019.
Recibido: 5 de marzo de 2019

Aceptado: 25 de agosto de 2019

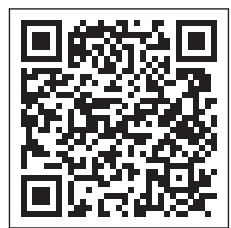

